\title{
General Framework using Affine Transformations to Formation Control Design
}

\author{
Lara Briñón Arranz* Alexandre Seuret** \\ Carlos Canudas de Wit ${ }^{* *}$ \\ * INRIA Rhône-Alpes, NeCS Team, Grenoble, France; (e-mail: \\ lara.brinon-arranz@inrialpes.fr). \\ ** CNRS, Department of Automatic Control, GIPSA-Lab, NeCS Team, \\ Grenoble, France (e-mail: alexandre.seuret, \\ carlos.canudas-de-wit@gipsa-lab.inpg.fr)
}

\begin{abstract}
This paper deals with the control of a fleet of non-linear systems representing AUVs (autonomous underwater vehicles). This paper proposes a novel framework which is able to express in a simple manner the control law for a larger class of formations, including time-varying formations. This has been produced by applying a sequence of affine transformations such as translations, rotations and scalings. A cooperative control law based on consensus algorithms is added to reach the same formation and to achieve the uniform distribution of all the agents along the formation. This is achieved taking into account the communication constraints using a cooperative control which includes the Laplacian matrix of the communication graph. Several simulations are provided to illustrate the convergence.
\end{abstract}

Keywords: Formation Control; Multi-agents Systems; Cooperative Control; Consensus.

\section{INTRODUCTION}

Cooperative control problems and multi-agent systems have received much attention in recent years (see OlfatiSaber et al. [2007]). This topic concerns an increasing number of engineering applications such as sensor networks, environmental monitoring among many others. This field includes the consensus algorithms for multi-agent systems Olfati-Saber et al. [2007], Olfati-Saber and Murray [2004], flocking Olfati-Saber [2006], distributed sensor networks Leonard et al. [2007], Ogren et al. [2004], Zhang and Leonard [2010], trajectory tracking and path following Kaminer et al. [1998], Frew et al. [2008], Encarnacao and Pascoal [2001] and autonomous systems as underwater and unmanned air vehicles (AUVs and UAVs) Fiorelli et al. [2003], Raffard et al. [2004].

A particularly relevant area deals with the formation control and the motion coordination, Leonard et al. [2007], Martínez et al. [2007], Ogren et al. [2002] and the reference therein. For instance, control laws have been provided to make a fleet of agents obtain circular and parallel formations, Leonard et al. [2007], Sepulchre et al. [2007] and other closed forms, Paley et al. [2008]. A relevant problem is now to relax these constraints and to consider timevarying formations. Two kind of transformations, translation and contraction, have been developed in Briñón et al. $[2009,2010]$ to stabilize a fleet of AUVs to a time-varying

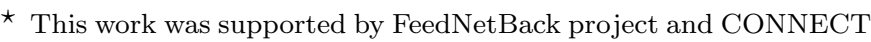
project. FeedNetBack is an EU STREP project FP7-ICT-2007-2 and the CONNECT project is funded by ANR (Agence Nationale de la Recherche), PSIROB06-174215
}

circle, in order to translate and contract the circular formation.

This paper provides a general framework based in the affine transformations to stabilize a formation of nonlinear multi-agent systems. Translation, contraction (scaling) and rotation are the three fundamental transformations of a formation (see Leonard and Fiorelli [2001]). These three main affine transformations, usually used in the fields of geometric and robotic control, are pertinent to express any kind of trajectory and closed curve in a matrix representation. The transformation of a fleet formation is pertinent to some applications where the agents should perform collaborative tasks requiring the formation to displace towards an a priori unknown direction and adjust to some particular form. For instance, in source seeking applications, the formation should displace in the source gradient direction and contract its size to adapt to the level curves of the source plume.

The general control law presented in this paper enables to track a reference velocity and to stabilize a time-varying curve. The formation control problem is tackled here from a collaborative point of view. A cooperative control law is developed that leads to the agents converging to the same formation which is defined by a closed curve expressed as a sequence of transformations. In the context of the source seeking for underwater vehicles, it is relevant to constrain the agents in an appropriate shape, to avoid unnecessary energy waist. Moreover, ensuring that the agents are uniformly distributed along the formation might be more adequate to produce efficient search motions. Therefore, an additional component of the control law is 
also added to achieved the uniform distribution of the agents along the time-varying formation. Moreover, this collaborative control law stands for the case of rangedependent graph, and provides some simulations showing the asymptotic convergence.

The paper is organized as follows. The following section presents the problem formulation introducing the affine transformation and the model of the agents. Section 3 exposes the main contribution of the article which deals with the general control law making the agents converge to a curve represented by a sequence of affine transformations and presents some particular cases and simulations. In section 4 a cooperative control is developed in order to stabilize the agents in the same formation.

\section{PROBLEM FORMULATION AND PRELIMINARIES}

\subsection{Model of Agents}

Consider the standard agent model commonly used in the literature to model AUVs restricted kinematics (Cochran and Krstic [2007], Leonard et al. [2007]). It corresponds to a kinematic unicycle fitting with model properties subject to a simple non-holonomic constraint. Consider a set of $N$ agents (vehicles), in which each agent $k=1, \ldots, N$ has the following constrained dynamics:

$$
\begin{aligned}
& \dot{x}_{k}=v_{k} \cos \theta_{k} \\
& \dot{y}_{k}=v_{k} \sin \theta_{k} \\
& \dot{v}_{k}=u_{1 k} \\
& \dot{\theta}_{k}=u_{2 k}
\end{aligned}
$$

where $\left(x_{k}, y_{k}\right)^{T}$ is the position vector of the agent $k, \theta_{k}$ the heading angle and $u_{1 k}, u_{2 k}$ are the control inputs. Note that $\mathbf{v} \in \mathbb{R}^{N}$ and $\theta \in \mathbb{R}^{N}$ represent the vector of all velocities and the vector of all headings respectively.

The objective is for the agents to follow different velocities and to stabilize to different formations in a cooperative way. In Sepulchre et al. [2007], a complex notation was introduced to formulate in a simple manner the circular formation control law. Here the vectorial notation (1) is employed and leads to a simple formulation of the control laws.

\subsection{Affine Transformations}

The three main transformations are the translation, the rotation and the scaling. To express these affine transformations, we need to introduce the homogeneous coordinates. For instance the homogeneous coordinates of a vector $z \in \mathbb{R}^{2}$ can simply be defined as the new vector $z^{H}=\left(z_{x}, z_{y}, 1\right)^{T}$. The affine transformations are introduced in order to express any trajectory in a matrix representation. The translation matrix for a vector $\mathbf{c}$ is $\mathbf{T}_{\mathbf{c}}=\left(\begin{array}{ccc}1 & 0 & c_{x} \\ 0 & 1 & c_{y} \\ 0 & 0 & 1\end{array}\right)$. The rotation by $\alpha$ is expressed as $\mathbf{R}_{\alpha}=\left(\begin{array}{ccc}\cos \alpha & -\sin \alpha & 0 \\ \sin \alpha & \cos \alpha & 0 \\ 0 & 0 & 1\end{array}\right)$ and $\mathbf{S}=\left(\begin{array}{ccc}s_{x} & 0 & 0 \\ 0 & s_{y} & 0 \\ 0 & 0 & 1\end{array}\right)$ is the non-uniform scaling matrix in homogeneous coordinates (see Foley et al. [1994]).
The main idea is to define any trajectory as a sequence of these transformations applied to a constant vector $\mathbf{r}_{0}$ expressed in the homogeneous coordinates framework. In the sequel, we consider affine transformations generated by a combination of the basic ones which can be defined as follows:

$$
\mathbf{G}=\prod_{i, j, k}^{I, J, K} \mathbf{S}^{i} \mathbf{R}_{\alpha}^{j} \mathbf{T}_{c}^{k}
$$

Note that the affine transformations are invertible, therefore $\mathbf{G}^{-1}$ exists. Thanks to previous definitions, $\mathbf{G}$ and $\mathbf{G}^{-1}$ are differentiable, if their parameters are timevarying. Note that the operator derivative and invertible are not commutative, therefore $(\dot{\mathbf{G}})^{-1} \neq\left(\mathbf{G}^{-1}\right)$.

\section{CONTROL DESIGN}

In previous section, the basic affine transformations have been defined. The objective is now to design control laws such that the fleet of agents converge to a curve defined by a sequence of affine transformations.

\subsection{Main Result}

Consider the system (1). The position vector of the agent $k$ in homogeneous coordinates is defined as $\mathbf{r}_{k}=\left(x_{k}, y_{k}, 1\right)^{T}$. The objective can now be expressed as:

$$
\mathbf{r}_{k}=\mathbf{G r}_{0}
$$

where $\mathbf{r}_{0}$ is a constant vector. The differentiation of previous equation leads to:

$$
\dot{\mathbf{r}}_{k}=\dot{\mathbf{G}} \mathbf{r}_{0}=\dot{\mathbf{G}} \mathbf{G}^{-1} \mathbf{r}_{k}
$$

Using the previous definitions of transformations, a control law is proposed in the following theorem:

Theorem 1. Let $\mathbf{G}$ be a twice differentiable matrix with bounded derivatives resulting from a sequence of affine transformations like in (2). Let $\kappa>0$ be a control parameter and the condition $v_{k} \neq 0$ is satisfied. Then the control law:

$$
\begin{aligned}
u_{1 k}= & -\kappa v_{k}+\frac{1}{v_{k}} \dot{\mathbf{r}}_{k}^{T} \dot{\mathbf{G}} \mathbf{G}^{-1} \dot{\mathbf{r}}_{k}+ \\
& +\frac{1}{v_{k}} \dot{\mathbf{r}}_{k}^{T}\left(\ddot{\mathbf{G}} \mathbf{G}^{-1}+\dot{\mathbf{G}}\left(\mathbf{G}^{-1}\right)+\kappa \dot{\mathbf{G}} \mathbf{G}^{-1}\right) \mathbf{r}_{k} \\
u_{2 k}= & \frac{1}{v_{k}^{2}} \dot{\mathbf{r}}_{k}^{T} \mathbf{R}^{* T} \dot{\mathbf{G}} \mathbf{G}^{-1} \dot{\mathbf{r}}_{k}+ \\
& +\frac{1}{v_{k}^{2}} \dot{\mathbf{r}}_{k}^{T} \mathbf{R}^{* T}\left(\ddot{\mathbf{G}} \mathbf{G}^{-1}+\dot{\mathbf{G}}\left(\mathbf{G}^{-1}\right)+\kappa \dot{\mathbf{G}} \mathbf{G}^{-1}\right) \mathbf{r}_{k}
\end{aligned}
$$

where the matrix $\mathbf{R}^{*}$ represents a rotation by $\frac{\pi}{2}$ but erasing the homogeneous coordinate of position vector such that:

$$
\mathbf{R}^{*}=\left(\begin{array}{ccc}
0 & -1 & 0 \\
1 & 0 & 0 \\
0 & 0 & 0
\end{array}\right)
$$

makes all the agents defined by (1) converge to the curve defined by the transformation $\mathbf{G}$ applied to the constant vector $\mathbf{r}_{0}$.

Proof. We analyze the convergence of the system to the curve defined by $\mathbf{G}$ using the following Lyapunov function:

$$
S(\mathbf{r}, \mathbf{v}, \theta)=\frac{1}{2} \sum_{k=1}^{N}\left\|\dot{\mathbf{r}}_{k}-\dot{\mathbf{G}} \mathbf{G}^{-1} \mathbf{r}_{k}\right\|^{2} \geq 0
$$


Note that when $S(\mathbf{r}, \mathbf{v}, \theta)=0$ the dynamics of system satisfy $\dot{\mathbf{r}}_{k}=\dot{\mathbf{G}} \mathbf{G}^{-1} \mathbf{r}_{k}$ which is the objective defined in (3). Evaluating the derivative of $S(\mathbf{r}, \mathbf{v}, \theta)$ along the solutions of system (1) leads to:

$$
\begin{aligned}
\dot{S}(\mathbf{r}, v, \psi)= & \sum_{k=1}^{N}\left(\ddot{\mathbf{r}}_{k}-\ddot{\mathbf{G}} \mathbf{G}^{-1} \mathbf{r}_{k}-\dot{\mathbf{G}}\left(\mathbf{G}^{-1}\right) \mathbf{r}_{k}-\dot{\mathbf{G}} \mathbf{G}^{-1} \dot{\mathbf{r}}_{k}\right)^{T} \\
& \cdot\left(\dot{\mathbf{r}}_{k}-\dot{\mathbf{G}} \mathbf{G}^{-1} \mathbf{r}_{k} \mathbf{r}_{k}\right)
\end{aligned}
$$

The second derivative of the position vector $\mathbf{r}_{k}$ expressed in homogeneous coordinates depends on the control input variables $u_{1 k}$ and $u_{2 k}$ :

$$
\ddot{\mathbf{r}}_{k}=\frac{\dot{v}_{k}}{v_{k}} \dot{\mathbf{r}}_{k}+\dot{\theta}_{k} \mathbf{R}^{*} \dot{\mathbf{r}}_{k}=\frac{u_{1 k}}{v_{k}} \dot{\mathbf{r}}_{k}+u_{2 k} \mathbf{R}^{*} \dot{\mathbf{r}}_{k}
$$

Considering the control law (4) and using the equation (6) the derivative of the Lyapunov function becomes:

$$
\dot{S}(\mathbf{r}, v, \psi)=-\kappa \sum_{k=1}^{N}\left\|\dot{\mathbf{r}}_{k}-\dot{\mathbf{G}} \mathbf{G}^{-1} \mathbf{r}_{k}\right\|^{2}=-2 \kappa S(\mathbf{r}, v, \theta) \leq 0
$$

Therefore $S(\mathbf{r}, \mathbf{v}, \theta)$ is a suitable Lyapunov function for this system. Then, system (1) asymptotically reaches the conditions $\dot{\mathbf{r}}_{k}=\dot{\mathbf{G}} \mathbf{G}^{-1} \mathbf{r}_{k}$ which describe a curve defined by $\mathbf{G}$.

Remark 2. The control law presented in the previous theorem has a singular point when $v_{k}=0$. The mathematical condition to avoid this situation is not straightforward obtained. The future works will be centered in a detailed analysis of this singular point. Nevertheless, the simulation results for the particular cases presented in the following subsections, show that if the initial velocities are positive for all the agents, and the trajectory represented by $\mathbf{G}$ varies slowly, the singular point is avoided.

This previous theorem present a general result to stabilize the agents to a curve defined by the matrix G. The following subsections present particular applications.

\subsection{Velocity Tracking}

First of all, we analyze the simplest case when the transformation is the identity matrix, that is, $\mathbf{G}=\mathbf{I}_{3}$. The objective becomes $\dot{\mathbf{r}}_{k}=0$. Thus, the control inputs become $u_{1 k}=-\kappa v_{k}$ and $u_{2 k}=0$. It is clear that in such situation, the objective only concerns the velocity and no constraint appears on the final position of the agents. These final positions depend on the initial condition of the agents. This fact is important to understand the following cases.

The trajectory tracking problem can be expressed as a transformation, specifically as a translation by the reference vector $\mathbf{r}_{r e f}=\left(x_{r e f}, y_{r e f}\right)$. Thus, the objective is:

$$
\mathbf{r}_{k}=\mathbf{T}_{\mathbf{r}_{r e f}} \mathbf{r}_{0}=\left(\begin{array}{ccc}
1 & 0 & x_{r e f} \\
0 & 1 & y_{r e f} \\
0 & 0 & 1
\end{array}\right)(0,0,1)^{T}=\mathbf{r}_{r e f}^{H}
$$

where $\mathbf{r}_{r e f}^{H}$ is the reference position vector in homogeneous coordinates. This objective expressed in the new formulation presented before leads with $\dot{\mathbf{r}}_{k}=\dot{\mathbf{T}}_{\mathbf{r}_{r e f}} \mathbf{T}_{\mathbf{r}_{r e f}}^{-1} \mathbf{r}_{k}=\dot{\mathbf{r}}_{r e f}^{H}$. Therefore, the system will be able to follow a reference

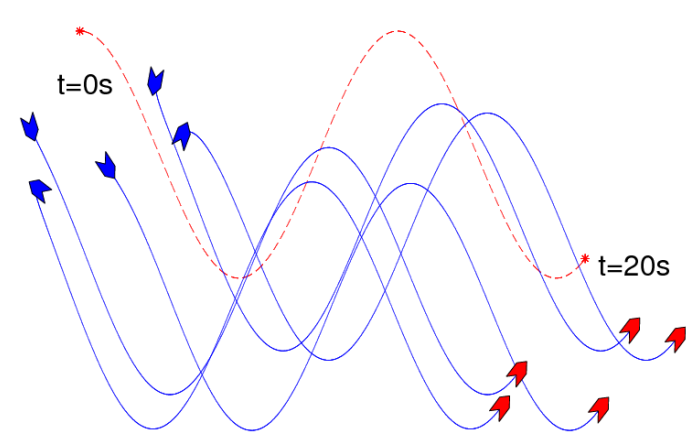

Fig. 1. Simulation of five agents governed by (7). The blue lines represent the trajectories of the agents following a reference velocity given by the red dashed line $\dot{\mathbf{r}}_{r e f}=\left(1,-2 \sin \frac{t}{2}\right)$. The figure shows two snapshots. The blue agents represent the initial conditions. The red ones represent the final state at $t=20 \mathrm{~s}$.

velocity. The control law dealing with this problem is presented in the following corollary:

Corollary 3. Let $\mathbf{r}_{\text {ref }}: \mathbb{R} \rightarrow \mathbb{R}^{2}$ a twice differentiable function with bounded first and second derivatives. Let $\kappa>0$ be a control parameter and the condition $v_{k} \neq 0$ is satisfied. Then the control law:

$$
\begin{aligned}
& u_{1 k}=-\kappa v_{k}+\frac{1}{v_{k}} \dot{\mathbf{r}}_{k}^{T}\left(\ddot{\mathbf{r}}_{r e f}^{H}+\kappa \dot{\mathbf{r}}_{r e f}^{H}\right) \\
& u_{2 k}=\frac{1}{v_{k}^{2}} \dot{\mathbf{r}}_{k}^{T} \mathbf{R}^{* T}\left(\ddot{\mathbf{r}}_{r e f}^{H}+\kappa \dot{\mathbf{r}}_{r e f}^{H}\right)
\end{aligned}
$$

makes all the agents defined by (1) follow the reference velocity $\dot{\mathbf{r}}_{\text {ref }}$.

Remark 4. Note that the agents follow the reference velocity and not the exact reference trajectory. This is not a trajectory tracking or path following problem. The formulation of the problem allows the agents to track the same velocity. We can define this as a velocity, as shown in Figure 1. Note that parallel motion problem analyzed in Sepulchre et al. [2007] loads to a straight line reference. Here, (7) is pertinent to consider more general references.

\subsection{Circular Trajectory}

A circular trajectory centered at 0 and of unit radius is described using the following parametrization in time:

$$
\begin{aligned}
& x(t)=\cos \left(\omega_{0} t\right) \\
& y(t)=\sin \left(\omega_{0} t\right)
\end{aligned}
$$

where $\omega_{0} \neq 0$ is the angular velocity of the rotation. The transformation matrix $\mathbf{G}$ becomes a time-varying rotation by the angle $\omega_{0} t$, that is, $\mathbf{G}=\mathbf{R}_{\omega_{0} t}$. In this case, the objective can be expressed as:

$$
\dot{\mathbf{r}}_{k}=\dot{\mathbf{R}}_{\omega_{0} t} \mathbf{R}_{\omega_{0} t}^{-1} \mathbf{r}_{k}=\omega_{0} \mathbf{R}^{*} \mathbf{r}_{k}
$$

Applying this objective to the control law (4) the following Corollary is obtained:

Corollary 5. Let $\omega_{0} \neq 0$ and $\kappa>0$ be two control parameters and the condition $v_{k} \neq 0$ is satisfied. Then the control law:

$$
\begin{aligned}
& u_{1 k}=-\kappa v_{k}+\kappa \frac{\omega_{0}}{v_{k}} \dot{\mathbf{r}}_{k}^{T} \mathbf{R}^{*} \mathbf{r}_{k} \\
& u_{2 k}=\omega_{0}+\kappa \frac{\omega_{0}}{v_{k}^{2}} \dot{\mathbf{r}}_{k}^{T} \mathbf{r}_{k}
\end{aligned}
$$


makes all the agents defined by (1) converge to a concentric circular trajectories centered at the origin and the direction of the rotation defined by the sign of $\omega_{0}$.

Remark 6. Once more, this formulation ensures that the agents converge to a circle. This leads to the same problem analyzed in Sepulchre et al. [2007]. However, no conditions on its radius is stated for now. As in the previous case, the initial conditions will influence the final radius. Note that the radius of the final circle of each agent satisfies $R_{k}=v_{k \infty} \omega_{0}$, where $v_{k \infty}$ is the final velocity of the agent $k$. This final value $v_{k \infty}$ is related to the initial conditions of the agent. Therefore, the radius depends of the initial conditions of the agents and thus, the agents converge to a circular trajectory with different radius.

Remark \%. Applying different sequences of transformations the agents can track a time-varying circular trajectory with a moving center and no constant radius. The results presented in Briñón et al. [2009, 2010] can be included in this new general formulation.

In order to achieve the same circle, and for more complex cases, the same formation, a cooperative control analysis is introduced in the following section.

\subsection{Not Circular Formations}

A particular case of the contraction or scaling of a circular trajectory can be consider when its radius depends on the agent position. In this way, many curves can be expressed by scaling of a circle. For example, a non-uniform timeinvariant scaling $\mathbf{S}=\left(\begin{array}{lll}a & 0 & 0 \\ 0 & b & 0 \\ 0 & 0 & 1\end{array}\right)$ where $a$ and $b$ are constant, defines an ellipse. In general, a scaling matrix depending on the position of the agent can be expressed as $\mathbf{S}_{k}=\left(\begin{array}{ccc}R_{k} & 0 & 0 \\ 0 & R_{k} & 0 \\ 0 & 0 & 1\end{array}\right)$ where the uniform scaling parameter

$R_{k}$ is a function leading to:

$$
\begin{aligned}
R_{k} & =R\left(\alpha_{k}\right) \\
\tan \alpha_{k} & =\frac{y_{k}}{x_{k}}
\end{aligned}
$$

The transformation matrices dealing with these problems are $\mathbf{G}=\mathbf{S R}_{\omega_{0} t}$ and $\mathbf{G}_{k}=\mathbf{S}_{k} \mathbf{R}_{\omega_{0} t}$ respectively. Applying the second formulation to the control law (4) the following Corollary is obtained:

Corollary 8. Let $R: \mathbb{R}^{2} \rightarrow \mathbb{R}$ a twice differentiable function with bounded first and second derivatives, as in (9). Let $\omega_{0} \neq 0$ and $\kappa>0$ be two control parameters and the condition $v_{k} \neq 0$ is satisfied. Then the control law:

$$
\begin{aligned}
u_{1 k}= & -\kappa v_{k}+\frac{\dot{R}_{k}}{R_{k}} v_{k}+\kappa \frac{\omega_{0}}{v_{k}} \dot{\mathbf{r}}_{k}^{T} \mathbf{R}^{*} \mathbf{r}_{k}+ \\
& +\frac{R_{k} \ddot{R}_{k}+\kappa R_{k} \dot{R}_{k}-\dot{R}_{k}^{2}}{v_{k} R_{k}^{2}} \dot{\mathbf{r}}_{k}^{T} \mathbf{r}_{k} \\
u_{2 k}= & \omega_{0}+\kappa \frac{\omega_{0}}{v_{k}^{2}} \dot{\mathbf{r}}_{k}^{T} \mathbf{r}_{k}+\frac{R_{k} \ddot{R}_{k}+\kappa R_{k} \dot{R}_{k}-\dot{R}_{k}^{2}}{v_{k} R_{k}^{2}} \dot{\mathbf{r}}_{k}^{T} \mathbf{r}_{k}(9 \mathrm{c})
\end{aligned}
$$

where $R_{k}=R\left(\alpha_{k}\right)$ makes all the agents defined by (1) converge to a curve defined by the function $R$ and the direction of the rotation defined by the sign of $\omega_{0}$.

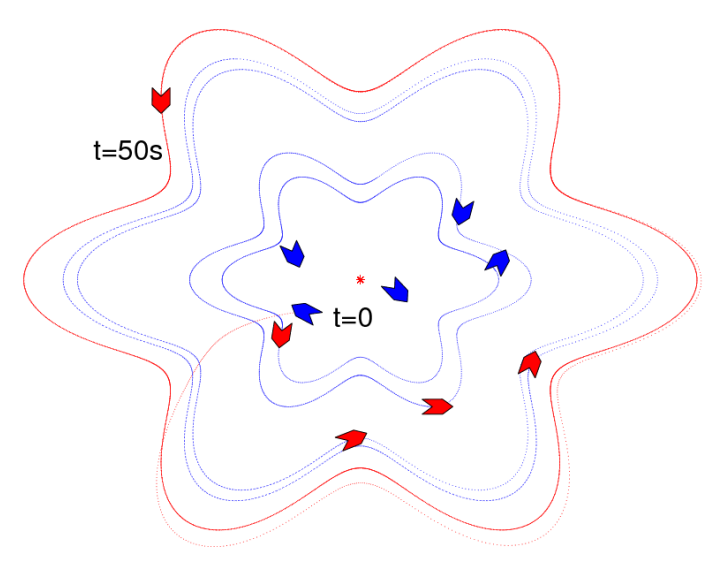

Fig. 2. Simulation of five agents governed by the control law (9) and the radius reference $R_{k}=\cos 6 \alpha_{k}+5$. The red line represents the trajectory of one agent and the blue ones represent the final curves achieves for each agent. The figure shows two snapshots. The blue agents represent the initial conditions. The red ones represent the final state $t=50 \mathrm{~s}$.

Remark 9. Note that one more time, the final trajectory of each agent is related to its final velocity which depends on the initial conditions. Therefore, each agent converge to a different curve but with the same shape and velocity of rotation, as in Figure 2.

\section{COOPERATIVE CONTROL DESIGN}

The general formulation presented in Theorem 1 allows to govern a group of agents to follow a reference velocity or to converge to a curve given by $\mathbf{G}$. Note that each agent converges to a different curve depending on the initial conditions but with the same shape. The aim to develop a cooperative control design deals with the stabilization of the same formation. This section presents collaborative control laws to obtain consensus on velocities and on the heading angles to reach a uniform distribution of the agents along the same formation.

\subsection{Preliminaries}

This paragraph presents some basic tools of graph theory. When an agent $k$ communicates with an agent $j$ both agents are called neighbors. The set of neighbors of agent $k$ is denoted by $\mathcal{N}_{k}$. The communication topology for the groups of agents can be represented by means of a graph $\mathcal{G}(V, E)$ where $V=\{1,2, \ldots, N\}$ is the set of vertices (agents) and $E=\left\{(k, j): j \in \mathcal{N}_{k}\right\}$ the set of edges (communication links) such that $(k, j) \in E$ if agent $k$ communicates with agent $j$. The Laplacian of $\mathcal{G}$ is the matrix $\mathbf{L}=\Delta-\mathbf{A}$. For an undirected graph, $(j$ is a neighbor of $k$ if and only if $k$ is a neighbor of $j$ ), the Laplacian matrix is symmetric positive semidefinite (see Olfati-Saber et al. [2007]).

\subsection{Consensus on Velocities for Circular Formation}

In the sequel, the cooperative control law to stabilize a circular formation using a consensus algorithm on the velocities is presented: 
Theorem 10. Let $\omega_{0} \neq 0, \kappa_{1}>0$ and $\kappa_{2}>0$ be three control parameters and the condition $v_{k} \neq 0$ is satisfied. Let $\mathcal{G}$ be the communication graph and $\mathbf{L}$ be the corresponding Laplacian matrix, where $\mathbf{L}_{k}$ represents its $k$ th row. Then the control law:

$$
\begin{aligned}
& u_{1 k}=-\kappa_{1} v_{k}+\kappa_{1} \frac{\omega_{0}}{v_{k}} \dot{\mathbf{r}}_{k}^{T} \mathbf{R}^{*} \mathbf{r}_{k}-\kappa_{2} \mathbf{L}_{k} \mathbf{v} \\
& u_{2 k}=\omega_{0}+\kappa_{1} \frac{\omega_{0}}{v_{k}^{2}} \dot{\mathbf{r}}_{k}^{T} \mathbf{r}_{k}
\end{aligned}
$$

makes all the agents defined by (1) converge to the same circular formation centered at the origin and the direction of the rotation defined by the sign of $\omega_{0}$. Moreover, if the communication graph $\mathcal{G}$ is strongly connected (see OlfatiSaber et al. [2007]), the radius of the circle is obtained through a consensus algorithm applied to the velocities of the agents.

Proof. The stability is analyzed by the composed Lyapunov function $V(\mathbf{r}, \mathbf{v}, \theta)=\kappa_{1} S(\mathbf{r}, \mathbf{v}, \theta)+\kappa_{2} Q(\mathbf{v})$ whose derivative is expressed as $\dot{V}(\mathbf{r}, \mathbf{v}, \theta)=\kappa_{1} \dot{S}(\mathbf{r}, \mathbf{v}, \theta)+$ $\kappa_{2} \dot{Q}(\mathbf{v})$. The quadratic form $Q(\mathbf{v})=\frac{1}{2} \mathbf{v}^{T} \mathbf{L v}$ reaches its minimum only when $\mathbf{v}=\mathbf{1} \beta$ where $\beta$ is constant, that is, when all agents turn around the same circle of radius $R=\beta \omega_{0}$.

Evaluating the derivative of $V(\mathbf{r}, \mathbf{v}, \theta)$ along the solutions of system (1) and using the equation (6):

$$
\begin{aligned}
\dot{V}(\mathbf{r}, v, \theta)= & \kappa_{1} \sum_{k=1}^{N}\left(\ddot{\mathbf{r}}_{k}-\omega_{0} \mathbf{R}^{*} \dot{\mathbf{r}}_{k}\right)^{T}\left(\dot{\mathbf{r}}_{k}-\omega_{0} \mathbf{R}^{*} \mathbf{r}_{k}\right)+ \\
& +\kappa_{2} \dot{\mathbf{v}}^{T} \mathbf{L} \mathbf{v} \\
= & \sum_{k=1}^{N} \dot{v}_{k}\left(\kappa_{1} v_{k}-\kappa_{1} \frac{\omega_{0}}{v_{k}} \dot{\mathbf{r}}_{k}^{T} \mathbf{R}^{*} \mathbf{r}_{k}+\kappa_{2} \mathbf{L}_{k} \mathbf{v}\right)+ \\
& +\kappa_{1} \sum_{k=1}^{N}\left(\omega_{0}-\dot{\theta}_{k}\right) \omega_{0} \dot{\mathbf{r}}_{k}^{T} \mathbf{r}_{k}
\end{aligned}
$$

Considering the control law (10) the previous equation leads to:

$$
\begin{aligned}
\dot{V}(\mathbf{r}, v, \theta)= & -\sum_{k=1}^{N}\left(\kappa_{1} v_{k}-\kappa_{1} \frac{\omega_{0}}{v_{k}} \dot{\mathbf{r}}_{k}^{T} \mathbf{R}^{*} \mathbf{r}_{k}-\kappa_{2} \mathbf{L}_{k} \mathbf{v}\right)^{2} \\
& -\kappa_{1} \sum_{k=1}^{N}\left(\frac{\omega_{0}}{v_{k}} \dot{\mathbf{r}}_{k}^{T} \mathbf{r}_{k}\right)^{2}
\end{aligned}
$$

Therefore $V(\mathbf{r}, \mathbf{v}, \theta)$ is a suitable Lyapunov function for this system and the agents converge to the same circular formation.

Remark 11. The work already cited, Sepulchre et al. [2007], deals with the circular formation problem. A formation control law is presented to stabilize a fixed circle. The agents have unit velocity and converge to a circle of radius $R=1 / \omega_{0}$. In our approach, the consensus algorithm provides the final radius.

Remark 12. Based on Olfati-Saber et al. [2007] and Sepulchre et al. [2008], using uniform connectivity properties, the collaborative control law in Theorem 10 also makes the agents converge to the same circular formation considering time-varying and switched communication graphs.

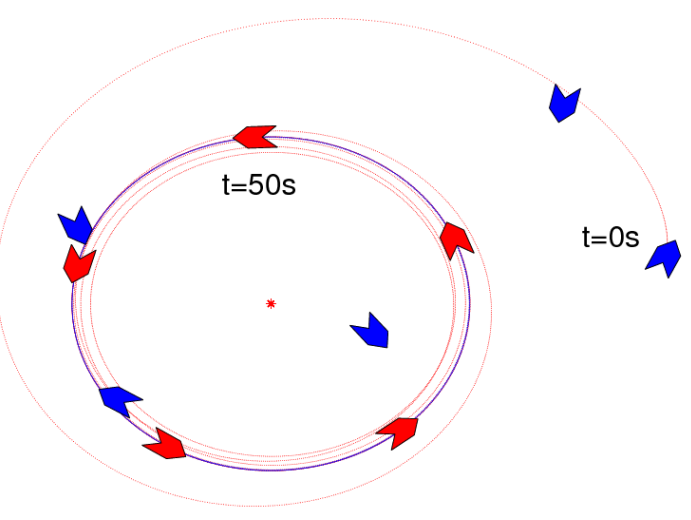

Fig. 3. Simulation of five agents governed by the control law (10). The same circular formation (blue line) with uniform distribution is stabilized. The red line represents the trajectory of one agent. The figure shows two snapshots. The blue agents represent the initial conditions. The red ones represent the final state $t=50 \mathrm{~s}$.

\subsection{Uniform Distribution for Circular Formation}

The following Corollary proposes an additional potential function whose minimum corresponds to the uniform distribution of the agents along the circular formation.

Corollary 13. Let $\omega_{0} \neq 0, \kappa_{1}>0, \kappa_{2}>0$, and $K>0$ be four control parameters. Let $\mathcal{G}$ be the communication graph and $\mathbf{L}$ be the corresponding Laplacian matrix. Then the control law:

$$
\begin{aligned}
& u_{1 k}=-\kappa_{1} v_{k}+\kappa_{1} \frac{\omega_{0}}{v_{k}} \dot{\mathbf{r}}_{k}^{T} \mathbf{R}^{*} \mathbf{r}_{k}-\kappa_{2} \mathbf{L}_{k} \mathbf{v} \\
& u_{2 k}=\omega_{0}+\kappa_{1} \frac{\omega_{0}}{v_{k}^{2}} \dot{\mathbf{r}}_{k}^{T} \mathbf{r}_{k}-\frac{\partial U}{\partial \theta_{k}}
\end{aligned}
$$

and

$$
U(\theta)=-\frac{K}{N} \sum_{m=1}^{\lfloor N / 2\rfloor} \frac{1}{2 m^{2}} \mathbf{B}_{m}^{T} \overline{\mathbf{L}} \mathbf{B}_{m}
$$

where $\lfloor N / 2\rfloor$ is the largest integer less than or equal to $N / 2$, the following equation $\overline{\mathbf{L}}=\mathbf{L} \otimes \mathbf{I}_{2}$ is satisfied ( $\otimes$ is the classical Kronecker product), and the matrix $\mathbf{B}_{m}=\left(\cos m \theta_{1}, \sin m \theta_{1}, \ldots, \cos m \theta_{N}, \sin m \theta_{N}\right)^{T}$ contains all the agents' headings, makes all the agents defined by (1) converge to a circular formation centered at the origin and the direction of the rotation defined by the sign of $\omega_{0}$. Moreover, if the communication graph $\mathcal{G}$ is $d_{0}$-circular (see Sepulchre et al. [2008], Briñón et al. [2010]), the radius of the circle is obtained through a consensus algorithm applying to the velocities of the agents and the uniform distribution of the agents along the circle is achieved.

Proof. The stability is analyzed by the composed Lyapunov function $V_{1}(\mathbf{r}, \mathbf{v}, \theta)=\kappa V(\mathbf{r}, \mathbf{v}, \theta)+U(\theta)$ whose derivative is expressed as $\dot{V}_{1}(\mathbf{r}, \mathbf{v}, \theta)=\dot{V}(\mathbf{r}, \mathbf{v}, \theta)+\nabla U(\theta)$. Based in the previous works of Sepulchre et al. [2007, 2008 ], the potential function $U(\theta)$ is invariant to rigid rotations. Therefore, using (11) the derivative of the Lyapunov function satisfies $\dot{V}_{1}(\mathbf{r}, \mathbf{v}, \theta) \leq 0$. 


\subsection{Towards Generalized Cooperative Control Laws}

Following the same approach applied to the circular formation, the objective is to provide a cooperative control added to the general control law (4) to stabilize the same formation. This is possible including consensus algorithms and potential functions to the general control law. In each case the consensus variable is different and this fact leads to several difficulties. The first result presented on the circle can be generalized to find the adequate variable for each formation, for exemple, in the translation of a circle, the consensus variable becomes $\tilde{v}=\left\|\dot{\mathbf{r}}_{k}-\dot{\mathbf{c}}_{\text {ref }}\right\|$ where $\mathbf{c}_{r e f}$ is a time-varying center reference.

\section{CONCLUSION AND FUTURES WORKS}

This paper provides a general framework to make a fleet of agents converge to formations defined by a sequence of affine transformations (translation, rotation and scaling). The control laws presented here make the agents converge to a time-varying velocity reference defined by the sequence of transformations G. Several cases have been presented. Moreover, a cooperative control law to achieve the circular formation and the uniform distribution of the agents along the circle is proposed. The consensus algorithm and the potential function added in this collaborative approach are designed taking into account the communication constraints between agents.

Future works will be focuss on finding the cooperative control algorithm to stabilize any formation expressed in the general framework.

\section{REFERENCES}

L. Briñón, A. Seuret, and C. Canudas. Translation control of a fleet circular formation of AUVs under finite communication range. Proceedings of the 48th IEEE Conference on Decision and Control, held jointly with the 28th Chinese Control Conference, 2009.

L. Briñón, A. Seuret, and C. Canudas. Contraction control of a fleet circular formation of AUVs under limited communication range. In Proceedings of the IEEE American Control Conference 2010, Baltimore, Maryland, USA, 2010.

J. Cochran and M. Krstic. Source seeking with a nonholonomic unicycle without position measurements and with tuning of angular velocity part I: Stability analysis. Proceedings of the 46th IEEE Conference on Decision and Control, 2007.

P. Encarnacao and A. Pascoal. Combined trajectory tracking and path following: an application to the coordinated control of autonomous marine craft. Proceedings of the 40th IEEE Conference on Decision and Control, 2001.

E. Fiorelli, P. Bhatta, N. E. Leonard, and I. Shulman. Adaptive sampling using feedback control of an autonomous underwater glider fleet. Proceedings of 13th Int. Symp. on Unmanned Untethered Submersible Technology (UUST), August 2003.

J. D. Foley, A. van Dam, S. K. Feiner, J. F. Hughes, and R. L. Phillips. Introduction to Computer Graphics. Addison-Wesley Publishing Co., 1994.

E. W. Frew, D. A. Lawrence, and S. Morris. Coordinated standoff tracking of moving targets using Lyapunov guidance vector fields. Journal of Guidance, Control, and Dynamics, 31(2):290-306, 2008.

I. Kaminer, A. Pascoal, E. Hallberg, and C. Silvestre. Trajectory tracking for autonomous vehicles: An integrated approach to guidance and control. Journal of Guidance, Control, and Dynamics, 21(1):29-38, 1998.

N. E. Leonard and E. Fiorelli. Virtual leaders, artifitial potentials and coordinated control of goups. Proceedings of 40st IEEE Conf. Decision and Control, 2001.

N. E. Leonard, D. A. Paley, F. Lekien, R. Sepulchre, D. M. Frantatoni, and R. E. Davis. Collective motion, sensor networks and ocean sampling. Proceedings of the IEEE, 95(1):48-74, January 2007.

S. Martínez, J. Cortés, and F. Bullo. Motion coordination with distributed information. IEEE Control Systems Magazine, 2007.

P. Ogren, M. Egerstedt, and X. Hu. A control Lyapunov function approach to multiagent coordination. IEEE Transactions on Automatic Control, 18(5), October 2002.

P. Ogren, E. Fiorelli, and N. Ehrich Leonard. Cooperative control of mobile sensor networks: Adaptive gradient climbing in a distributed environment. IEEE Transactions on Automatic Control, 49, August 2004.

R. Olfati-Saber. Flocking for multi-agent dynamic systems: Algorithms and theory. IEEE Transactions on Automatic Control, 51(3):401420, March 2006.

R. Olfati-Saber and R. M. Murray. Consensus problems in networks of agents with switching topology and timedelays. IEEE Transactions on Automatic Control, 49(9): 1520-1533, September 2004.

R. Olfati-Saber, J. A. Fax, and R. M. Murray. Consensus and cooperation in networked multi-agent systems. Proceedings of the IEEE, (1), January 2007.

D. A. Paley, N. E. Leonard, and R. Sepulchre. Stabilization of symmetric formations to motion around convex loops. Systems \& Control Letters, 57:209-215, 2008.

R. L. Raffard, C. J. Tomlin, and S. P. Boyd. Distributed optimization for cooperative agents: Application to formation flight. 43rd IEEE Conference on Decision and Control, 2004.

R. Sepulchre, D. A. Paley, and N. E. Leonard. Stabilization of planar collective motion: All-to-all communication. IEEE Transactions on Automatic Control, 2007.

R. Sepulchre, D. A. Paley, and N. E. Leonard. Stabilization of planar collective motion with limited communication. IEEE Transactions on Automatic Control, 53:706-719, 2008.

F. Zhang and N. E. Leonard. Cooperative filters and control for cooperative exploration. IEEE Transactions on Automatic Control, 55:650 - 663, 2010. 